\title{
MGLM: An R Package for Multivariate Categorical Data Analysis
}

\author{
by Juhyun Kim, Yiwen Zhang, Joshua Day, Hua Zhou
}

\begin{abstract}
Data with multiple responses is ubiquitous in modern applications. However, few tools are available for regression analysis of multivariate counts. The most popular multinomial-logit model has a very restrictive mean-variance structure, limiting its applicability to many data sets. This article introduces an R package MGLM, short for multivariate response generalized linear models, that expands the current tools for regression analysis of polytomous data. Distribution fitting, random number generation, regression, and sparse regression are treated in a unifying framework. The algorithm, usage, and implementation details are discussed.
\end{abstract}

\section{Introduction}

Multivariate categorical data arises in many fields, including genomics, image analysis, text mining, and sports statistics. The multinomial-logit model (Agresti, 2002, Chapter 7) has been the most popular tool for analyzing such data. However, it is limiting due to its specific mean-variance structure and the strong assumption that the counts are negatively correlated. Models that address over-dispersion relative to a multinomial distribution and incorporate positive and/or negative correlation structures would offer greater flexibility for analysis of polytomous data.

In this article, we introduce an R package MGLM, short for multivariate response generalized linear models. The MGLM package provides a unified framework for random number generation, distribution fitting, regression, hypothesis testing, and variable selection for multivariate response generalized linear models, particularly four models listed in Table 1. These models considerably broaden the class of generalized linear models (GLM) for analysis of multivariate categorical data.

MGLM overlaps little with existing packages in $\mathrm{R}$ and other softwares. The standard multinomiallogit model is implemented in several R packages (Venables and Ripley, 2002) with VGAM (Yee, 2010, 2015 , 2017) being the most comprehensive. We include the classical multinomial-logit regression model in MGLM not only for completeness, but also to complement it with various penalty methods for variable selection and regularization. If invoked by the group penalty, MGLM is able to perform variable selection at the predictor level for easier interpretation. This is different from the elastic net penalized multinomial-logit model implemented in glmnet (Friedman et al., 2010), which does selection at the matrix entry level. Although MGLM focuses on regression, it also provides distribution fitting and random number generation for the models listed in Table 1. VGAM and dirmult (Tvedebrink, 2010) packages can estimate the parameters of the Dirichlet-multinomial (DM) distribution using Fisher's scoring and Newton's method respectively. As indicated in the manual (Yee, 2017), the convergence of Fisher's scoring method may be slow due to the difficulty in evaluating the expected information matrix. Further the Newton's method is unstable as the log-likelihood function may be non-concave. As explained later, MGLM achieves both stability and efficiency via a careful algorithmic design. In SAS, PROC LOGISTIC can fit multinomial-logit model. In Matlab, the mnrfit function fits multinomiallogit regression. Alternative link functions (probit, loglog, complementary loglog) are implemented only for ordinal responses. Other regression models in Table 1 are not implemented in either SAS or Matlab.

There are some limitations to the MGLM. First, MGLM only handles nominal responses; ordinal responses are not incorporated in current implementation. MGLM also does not allow covariates to take a different value for each category, which can be useful in applications such as modeling consumer choice among a discrete number of products (Yee, 2015, Chapter 14). Lastly, current implementation of MGLM does not permit parameter constraints.

MGLM provides standard errors for all estimates, reports significance of regression covariates based on the Wald test (default) or the likelihood ratio test (LRT), and outputs the AIC (Akaike information criterion) and BIC (Bayesian information criterion) of the fitted model to aid model choice by users. Model selection via regularization is automated by computing the solution path on a grid of tuning parameter values and then reporting the regularized estimate with minimal BIC. With large data sets in mind, we pay particular attention to computational efficiency. For numerical examples in this article, we report the run times whenever possible. The results are obtained on a laptop with Intel Core i7 CPU @ 2.9GHz and 16G memory using MGLM 0.1.0 under R 3.4.3. 


\begin{tabular}{llcl}
\hline Model & No. regress. param. & Correlation & MGLM code \\
\hline Multinomial & $p(d-1)$ & Negative & dist='MN' \\
Dirichlet-multinomial & $p d$ & Negative & dist=' DM' \\
Negative multinomial & $p(d+1)$ or $p d+1$ & Positive & dist='NegMN' \\
Gen. Dirichlet-multinomial & $2 p(d-1)$ & Negative and positive & dist='GDM' \\
\hline
\end{tabular}

Table 1: Multivariate generalized linear model implemented in the MGLM package. $d$ is the number of categories and $p$ is the number of predictors in the regression model.

\section{Multivariate generalized linear models (MGLM)}

This section details the models implemented in MGLM. Table 1 summarizes the multivariate models implemented in the R package. They are multivariate analogs of binomial, beta-binomial, and negative binomial models. For each model, we specify the probability mass function of the response vector $\mathbf{y}$, the link function that relates distribution parameters to covariates, and the log-likelihood function of a finite sample. We start from the classical multinomial-logit model.

\section{Multinomial (MN) model}

The probability mass function of a $d$ dimensional multinomial sample $\mathbf{y}=\left(y_{1}, \ldots, y_{d}\right)^{T}$ with batch size $m=\sum_{j=1}^{d} y_{j}$ and parameter $\boldsymbol{p}=\left(p_{1}, \ldots, p_{d}\right)$ is

$$
f(\mathbf{y} \mid \boldsymbol{p})=\left(\begin{array}{c}
m \\
\mathbf{y}
\end{array}\right) \prod_{j=1}^{d} p_{j}^{y_{j}} .
$$

The parameter $\boldsymbol{p}$ is linked to the covariate vector $\mathbf{x} \in \mathbb{R}^{p}$ via the multinomial-Poisson transformation (Baker, 1994; Lang, 1996)

$$
p_{j}=\frac{e^{\mathbf{x}^{T} \boldsymbol{\beta}_{j}}}{\sum_{j^{\prime}} e^{\mathbf{x}^{\mathrm{T}} \boldsymbol{\beta}_{j^{\prime}}}}, \quad j=1, \ldots, d,
$$

where $\beta_{1}, \ldots, \boldsymbol{\beta}_{d} \in \mathbb{R}^{p}$ are the regression coefficients. For identifiability, we constrain $\boldsymbol{\beta}_{d}=\mathbf{0}$ and only estimate $\beta_{1}, \ldots, \beta_{d-1}$, which are collected in the regression coefficient matrix $\boldsymbol{B} \in \mathbb{R}^{p \times(d-1)}$. Given independent observations $\left(\mathbf{y}_{i}, \mathbf{x}_{i}\right), i=1, \ldots, n$, the log-likelihood is

$$
\ell(\boldsymbol{B})=\sum_{i=1}^{n} \sum_{j=1}^{d} y_{i j}\left(\mathbf{x}_{i}^{T} \boldsymbol{\beta}_{j}-\ln \sum_{j^{\prime}=1}^{d} e^{\mathbf{x}_{i}^{T} \boldsymbol{\beta}_{j^{\prime}}}\right)+\sum_{i=1}^{n} \ln \left(\begin{array}{c}
m_{i} \\
\mathbf{y}_{i}
\end{array}\right) .
$$

Because the log-sum-exponential mapping $\left(\eta_{1}, \ldots, \eta_{d}\right)^{T} \mapsto \ln \sum_{j} e^{\eta_{j}}$ is convex (Boyd and Vandenberghe, 2004, p72), the log-likelihood function is concave. This nice feature makes maximum likelihood estimation relatively easy for multinomial-logit model. Unfortunately, convexity is lost in other models listed in Table 1.

\section{Dirichlet-multinomial (DM) model}

The mean-variance structure of the MN model does not allow over-dispersion, which is common in real data. DM distribution models the probability parameter $p$ in the MN model by a Dirichlet distribution. The probability mass of a $d$-category count vector $\mathbf{y}$ over $m=\sum_{j} y_{j}$ trials under DM with parameter $\boldsymbol{\alpha}=\left(\alpha_{1}, \ldots, \alpha_{d}\right), \alpha_{j}>0$ and proportion vector $\mathbf{p} \in \Delta_{d}=\left\{\left(p_{1}, \ldots, p_{d}\right): p_{j} \geq 0, \sum_{j} p_{j}=1\right\}$ is 


$$
\begin{aligned}
f(\mathbf{y} \mid \boldsymbol{\alpha}) & =\int_{\Delta_{d}}\left(\begin{array}{c}
m \\
\mathbf{y}
\end{array}\right) \prod_{j} p_{j}^{y_{j}} \frac{\Gamma\left(\sum_{j} \alpha_{j}\right)}{\prod_{j} \Gamma\left(\alpha_{j}\right)} \prod_{j} p_{j}^{\alpha_{j}-1} d p \\
& =\left(\begin{array}{c}
m \\
\mathbf{y}
\end{array}\right) \prod_{j=1}^{d} \frac{\Gamma\left(\alpha_{j}+y_{j}\right)}{\Gamma\left(\alpha_{j}\right)} \frac{\Gamma\left(\sum_{j} \alpha_{j}\right)}{\Gamma\left(\sum_{j} \alpha_{j}+\sum_{j} y_{j}\right)} \\
& =\left(\begin{array}{c}
m \\
\mathbf{y}
\end{array}\right) \frac{\prod_{j=1}^{d}\left(\alpha_{j}\right)_{y_{j}}}{\left(\sum_{j} \alpha_{j}\right)_{m}},
\end{aligned}
$$

where $(a)_{k}=a(a+1) \cdots(a+k-1)$ for nonnegative integer $k$ denotes the rising factorial. The last equality is due to the identity $\Gamma(a+k) / \Gamma(a)=(a)_{k}$ (Graham et al., 1994). Because the data $y_{j}$ and parameter $\alpha_{j}$ are intertwined in the gamma (or rising factorial) terms and do not factorize, the DM distribution does not belong to the exponential family. To incorporate covariates, the inverse link function

$$
\alpha_{j}=e^{\mathbf{x}^{T} \boldsymbol{\beta}_{j}}, \quad j=1, \ldots, d
$$

relates the parameter $\alpha=\left(\alpha_{1}, \ldots, \alpha_{d}\right)$ of the DM distribution to the covariates $\mathbf{x}$. The log-likelihood for independent data points $\left(\mathbf{y}_{i}, \mathbf{x}_{i}\right), i=1, \ldots, n$, takes the form

$$
\ell(\boldsymbol{B})=\sum_{i=1}^{n} \sum_{j=1}^{d} \sum_{k=0}^{y_{i j}-1} \ln \left(e^{\mathbf{x}_{i}^{T} \boldsymbol{\beta}_{j}}+k\right)-\sum_{i=1}^{n} \sum_{k=0}^{m_{i}-1} \ln \left(\sum_{j=1}^{d} e^{\mathbf{x}_{i}^{T} \boldsymbol{\beta}_{j}}+k\right)+\sum_{i=1}^{n} \ln \left(\begin{array}{l}
m_{i} \\
\mathbf{y}_{i}
\end{array}\right)
$$

with $\boldsymbol{B}=\left(\boldsymbol{\beta}_{1}, \ldots, \boldsymbol{\beta}_{d}\right) \in \mathbb{R}^{p \times d}$ collecting all regression coefficients. The log-likelihood, as a difference of two convex terms, is not concave in general.

\section{Negative multinomial (NegMN) model}

The counts $Y_{j}$ in both MN and DM models are negatively correlated, restricting their use for counts with positive correlation. The NegMN distribution provides a natural model for positively correlated count data. The probability mass of a count vector $\mathbf{y}$ under a NegMN distribution with parameter $\left(p_{1}, \ldots, p_{d+1}, \phi\right), \sum_{j=1}^{d+1} p_{j}=1, p_{j}, \phi>0$, is

$$
f(\mathbf{y} \mid \boldsymbol{p}, \phi)=\left(\begin{array}{c}
\phi+m-1 \\
m
\end{array}\right)\left(\begin{array}{l}
m \\
\mathbf{y}
\end{array}\right) \prod_{j=1}^{d} p_{j}^{y_{j}} p_{d+1}^{\phi}=\frac{(\phi)_{m}}{m !}\left(\begin{array}{c}
m \\
\mathbf{y}
\end{array}\right) \prod_{j=1}^{d} p_{j}^{y_{j}} p_{d+1^{\prime}}^{\phi}
$$

where $\left(\begin{array}{c}\phi+m-1 \\ m\end{array}\right)=\frac{(\phi)_{m}}{m !}$ is the general binomial coefficient. The parameter $\phi$ and the data $m$ do not factorize. Therefore, NegMN does not belong to the exponential family when $\phi$ is unknown. We use the inverse link functions

$$
p_{j}=\frac{e^{\mathbf{x}^{T} \boldsymbol{\alpha}_{j}}}{1+\sum_{j=1}^{d} e^{\mathbf{x}^{T} \boldsymbol{\alpha}_{j}}}, 1 \leq j \leq d, \quad p_{d+1}=\frac{1}{1+\sum_{j=1}^{d} e^{\mathbf{x}^{T} \boldsymbol{\alpha}_{j}}}, \quad \phi=e^{\mathbf{x}^{T} \boldsymbol{\beta}}
$$

to relate the covariates $\mathbf{x}$ to distribution parameter $\left(p_{1}, \ldots, p_{d+1}, \phi\right)$. Let $\boldsymbol{B}=\left(\boldsymbol{\alpha}_{1}, \ldots, \boldsymbol{\alpha}_{d}, \boldsymbol{\beta}\right) \in \mathbb{R}^{p \times(d+1)}$ collect all the regression coefficients. Given $n$ independent data points $\left(\mathbf{y}_{i}, \mathbf{x}_{i}\right), i=1, \ldots, n$, the $\log$ likelihood is

$$
\begin{aligned}
\ell(\boldsymbol{B})= & \sum_{i=1}^{n} \sum_{k=0}^{m_{i}-1} \ln \left(e^{\mathbf{x}_{i}^{T} \boldsymbol{\beta}}+k\right)-\sum_{i=1}^{n}\left(e^{\mathbf{x}_{i}^{T} \boldsymbol{\beta}}+m_{i}\right) \ln \left(\sum_{j=1}^{d} e^{\mathbf{x}_{i}^{T} \boldsymbol{\alpha}_{j}}+1\right) \\
& +\sum_{i=1}^{n} \sum_{j=1}^{d} y_{i j} \mathbf{x}_{i}^{T} \boldsymbol{\alpha}_{j}-\sum_{i=1}^{n} \sum_{j=1}^{d} \ln y_{i j} !,
\end{aligned}
$$

which in general is not concave in $\alpha_{j}$ and $\beta$.

In some applications the over-dispersion parameter $\phi$ may not depend on the covariates $\mathbf{x}$. MGLM offers the option to model the responses $\mathbf{y}_{i}$ to share a common dispersion parameter $\phi$, without linking it to the covariates. In this case, the log-likelihood is 


$$
\begin{aligned}
\ell\left(\boldsymbol{\alpha}_{1}, \ldots, \boldsymbol{\alpha}_{d}, \phi\right)= & \sum_{i=1}^{n} \sum_{k=0}^{m_{i}-1} \ln (\phi+k)-\sum_{i=1}^{n}\left(\phi+m_{i}\right) \ln \left(\sum_{j=1}^{d} e^{\mathbf{x}_{i}^{T} \boldsymbol{\alpha}_{j}}+1\right) \\
& +\sum_{i=1}^{n} \sum_{j=1}^{d} y_{i j} \mathbf{x}_{i}^{T} \boldsymbol{\alpha}_{j}-\sum_{i=1}^{n} \sum_{j=1}^{d} \ln y_{i j} !
\end{aligned}
$$

The model size is $p d+1$ and MGLM outputs the estimates $\left(\hat{\boldsymbol{\alpha}}_{1}, \ldots, \hat{\boldsymbol{\alpha}}_{d}, \hat{\phi}\right)$ and their standard errors.

\section{Generalized Dirichlet-multinomial (GDM) model}

In the previous three models, the multivariate counts have either pairwise negative correlation ( $\mathrm{MN}$ and $\mathrm{DM}$ ) or pairwise positive correlation (NegMN). It is possible to relax these restrictions by choosing a more flexible mixing distribution for the probability vector $p$ in MN model. Connor and Mosimann (1969) suggest a generalization of the Dirichlet distribution that meets this challenge. The resulting admixed distribution, called the GDM distribution, provides a flexible model for multivariate categorical data with a general correlation structure (Bouguila, 2008; Zhou and Lange, 2010).

The probability mass of a count vector $\mathbf{y}$ over $m=\sum_{j} y_{j}$ trials under GDM with parameter $(\boldsymbol{\alpha}, \boldsymbol{\beta})=\left(\alpha_{1}, \ldots, \alpha_{d-1}, \beta_{1}, \ldots, \beta_{d-1}\right), \alpha_{j}, \beta_{j}>0$, is

$$
\begin{aligned}
f(\mathbf{y} \mid \boldsymbol{\alpha}, \boldsymbol{\beta}) & =\left(\begin{array}{c}
m \\
\mathbf{y}
\end{array}\right) \prod_{j=1}^{d-1} \frac{\Gamma\left(\alpha_{j}+y_{j}\right)}{\Gamma\left(\alpha_{j}\right)} \frac{\Gamma\left(\beta_{j}+z_{j+1}\right)}{\Gamma\left(\beta_{j}\right)} \frac{\Gamma\left(\alpha_{j}+\beta_{j}\right)}{\Gamma\left(\alpha_{j}+\beta_{j}+z_{j}\right)} \\
& =\left(\begin{array}{c}
m \\
\mathbf{y}
\end{array}\right) \prod_{j=1}^{d-1} \frac{\left(\alpha_{j}\right)_{y_{j}}\left(\beta_{j}\right)_{z_{j+1}}}{\left(\alpha_{j}+\beta_{j}\right)_{z_{j}}},
\end{aligned}
$$

where $z_{j}=\sum_{k=j}^{d} y_{k}$. Again it is clear that the GDM distribution does not belong to the exponential family, since the parameter $\alpha_{j}, \beta_{j}$ and data $y_{j}, z_{j}$ do not factorize.

We propose the inverse link functions

$$
\alpha_{j}=e^{\mathbf{x}^{T} \boldsymbol{\alpha}_{j}}, \quad \beta_{j}=e^{\mathbf{x}^{T} \boldsymbol{\beta}_{j}}, \quad 1 \leq j \leq d-1,
$$

to relate the covariates $\mathbf{x}$ and parameter $\left(\alpha_{1}, \ldots, \alpha_{d-1}, \beta_{1}, \ldots, \beta_{d-1}\right)$ of the GDM distribution (1). Let $\boldsymbol{B}=\left(\boldsymbol{\alpha}_{1}, \ldots, \boldsymbol{\alpha}_{d-1}, \boldsymbol{\beta}_{1}, \ldots, \boldsymbol{\beta}_{d-1}\right) \in \mathbb{R}^{p \times 2(d-1)}$ collect all the regression coefficients. Given $n$ independent data points $\left(\mathbf{y}_{i}, \mathbf{x}_{i}\right)$, the log-likelihood is

$$
\begin{aligned}
\ell(\boldsymbol{B})= & \sum_{i=1}^{n} \sum_{j=1}^{d-1}\left[\sum_{k=0}^{y_{i j}-1} \ln \left(e^{\mathbf{x}_{i}^{T} \boldsymbol{\alpha}_{j}}+k\right)+\sum_{k=0}^{z_{i, j+1}-1} \ln \left(e^{\mathbf{x}_{i}^{T} \boldsymbol{\beta}_{j}}+k\right)\right. \\
& \left.-\sum_{k=0}^{z_{i j}-1} \ln \left(e^{\mathbf{x}_{i}^{T} \boldsymbol{\alpha}_{j}}+e^{\mathbf{x}_{i}^{T} \boldsymbol{\beta}_{j}}+k\right)\right]+\sum_{i=1}^{n} \ln \left(\begin{array}{c}
m_{i} \\
\mathbf{y}_{i}
\end{array}\right) .
\end{aligned}
$$

Again the log-likelihood is not concave in general.

\section{Which model to use?}

When there are no covariates, multinomial model is a special case of the DM models, which in turn is a sub-model of the GDM model. That is, MN $\subset \mathrm{DM} \subset \mathrm{GDM}$. The distribution fitting function MGLMfit reports the $p$-value of the LRT for comparing the fitted model with the most commonly used multinomial model. NegMN model does not have such a relationship with any of the other three models. Therefore, no LRT is performed when dist="NegMN" in the distribution fitting function MGLMfit.

For regression, there is no nesting structure among the models in Table 1 . The regression function MGLMreg outputs AIC and BIC of the fitted model to aid users in choosing an appropriate regression model for their data. 


\section{Standard errors and testing}

Standard errors for both distribution fitting (MGLMfit) and regression estimates (MGLMreg) are calculated based on the observed information matrix, as it provides a reasonable approximation to the expected information matrix and is even preferred as argued by Efron and Hinkley (1978).

Unlike regression for univariate responses, the MGLM regression parameter is a matrix $\boldsymbol{B}=$ $\left(\boldsymbol{\beta}_{1}, \ldots, \boldsymbol{\beta}_{d_{e}}\right) \in \mathbb{R}^{p \times d_{e}}$ with each row $\boldsymbol{B}_{k}$. corresponding to the effects of one predictor. Here $d_{e}=d-1$ $(\mathrm{MN}), d(\mathrm{DM}), d+1(\mathrm{NegMN})$, or $2(d-1)(\mathrm{GDM})$. Therefore, the hypotheses for testing the significance of the $k$-th covariate are:

$$
H_{0}:\left\|\boldsymbol{B}_{k, \cdot}^{T}\right\|_{2}=0 \quad \text { vs } \quad H_{a}:\left\|\boldsymbol{B}_{k, \cdot}^{T}\right\| \neq 0 .
$$

By default, MGLMreg assesses the significance of each predictor by the Wald test. Let

$$
\widehat{\boldsymbol{\Sigma}}=\boldsymbol{I}_{\text {obs }}^{-1}(\widehat{\boldsymbol{B}})=\left[-\nabla^{2} \ell(\widehat{\boldsymbol{B}})\right]^{-1} \in \mathbb{R}^{p d_{e} \times p d_{e}}
$$

be the inverse of the observed information matrix at the maximum likelihood estimate (MLE) $\widehat{\boldsymbol{B}}$. Then the Wald statistic is computed as

$$
W_{k}=\widehat{\boldsymbol{B}}_{k,} \widehat{\boldsymbol{\Sigma}}_{k, k} \widehat{\boldsymbol{B}}_{k, \cdot}^{T}
$$

where $\widehat{\boldsymbol{\Sigma}}_{k, k}$ is the sub-matrix of $\widehat{\boldsymbol{\Sigma}}$ obtained by selecting rows and columns corresponding to the entries of $\boldsymbol{B}_{k, .} . W_{k}$ is asymptotically distributed as a chi-square distribution with $d_{e}$ degrees of freedom under the null distribution, yielding the $p$-values reported by MGLMreg. Users can also easily invoke the LRT by calling MGLMreg with option LRT=TRUE. LRT requires more computation than Wald test as it needs to perform MLE on $p$ sub-models.

\section{Regularization}

The number of parameters, $p d_{e}$, in the multivariate response models can be unwieldy with a moderate to large number of predictors. When the sample size $n$ is limited or even smaller than $p d_{e}$, regularization is necessary for variable selection, model interpretation, and improving the risk property of the estimate. In general, the MGLMsparsereg function solves the regularized problem

$$
\min _{\boldsymbol{B}}-\ell(\boldsymbol{B})+J(\boldsymbol{B}),
$$

where $\ell$ is the log-likelihood function and $J$ is a penalty term. The choice of $J$ depends on specific applications. We implemented three classes of penalties. Below, $\mathcal{S}$ is an index set for the set of predictors subject to regularization, which can be selectively specified by the users.

In elementwise regularization (penalty= ' sweep '),

$$
J(\boldsymbol{B})=\sum_{k \in \mathcal{S}} \sum_{j=1}^{d_{e}} P_{\eta}\left(\left|\beta_{k j}\right|, \lambda\right),
$$

where $P$ is a scalar penalty function, $\lambda$ is the penalty tuning constant, and $\eta$ is a parameter indexing member of a penalty family. Choices of the penalty families include: power family (Frank and Friedman, 1993), where $P_{\eta}(|x|, \lambda)=\lambda|x|^{\eta}, \eta \in(0,2]$, and in particular lasso (Tibshirani, 1996) $(\eta=1)$ and ridge $(\eta=2)$; elastic net (Zou and Hastie, 2005), where $P_{\eta}(|x|, \lambda)=\lambda\left[(\eta-1) x^{2} / 2+(2-\eta)|x|\right], \eta \in$ $[1,2] ; \operatorname{SCAD}$ (Fan and Li, 2001), where $\partial / \partial|x| P_{\eta}(|x|, \lambda)=\lambda\left\{1_{\{|x| \leq \lambda\}}+(\eta \lambda-|x|)_{+} /(\eta-1) \lambda 1_{\{|x|>\lambda\}}\right\}$, $\eta>2$; and MC+ penalty (Zhang, 2010), where $P_{\eta}(|x|, \lambda)=\left\{\lambda|x|-x^{2} /(2 \eta)\right\} 1_{\{|x|<\eta \lambda\}}+0.5 \lambda^{2} \eta 1_{\{|x| \geq \eta \lambda\}}$. The special case of elastic net for the multinomial-logit model is also implemented in the popular glmnet package by Friedman et al. (2010) using coordinate descent algorithm. MGLM implements a generic accelerated proximal gradient method that applies to the following two penalties too.

In groupwise regularization (penal ty= ' group'),

$$
J(B)=\lambda \sum_{k \in \mathcal{S}}\left\|\boldsymbol{B}_{k,}\right\|_{2}=\lambda \sum_{k \in \mathcal{S}}\left(\sum_{j=1}^{d_{e}} \beta_{k j}^{2}\right)^{1 / 2} .
$$

The group penalty (Yuan and Lin, 2006; Meier et al., 2008) achieves variable selection at the predictor level and leads to a more interpretable model. 
Shrinkage and sparsity in terms of the rank of $\boldsymbol{B}$ is achieved by the nuclear norm regularization (penalty= 'nuclear')

$$
J(\boldsymbol{B})=\|\boldsymbol{B}\|_{*}=\lambda \sum_{i} \sigma_{i}(\boldsymbol{B}),
$$

where $\sigma_{i}(\boldsymbol{B})$ 's are the singular values of the matrix $\boldsymbol{B}$. The nuclear norm $\|\boldsymbol{B}\|_{*}$ is a suitable convex relaxation of the rank of a matrix parameter. It encourages low rank of the parameter matrix estimate and has been successfully employed in the matrix completion problem (Mazumder et al., 2010), regression with matrix covariates (Zhou and Li, 2014) and predicting at-bat results in baseball (Powers et al.).

The wrapper function MGLMtune facilitates the tuning procedure by performing the regularized estimation over a grid of 30 (default) tuning parameter values using warm start and reports the optimal tuning parameter according to BIC. Users can also supply their own grid points.

\section{Optimization algorithms and implementation}

As the DM, NegMN, and GDM distributions do not belong to the exponential family, the usual iteratively reweighted least squares method for maximum likelihood estimation of GLM does not apply. The main issue lies in the difficulty of calculating the expected information matrix, which involves evaluating numerous tail probabilities of the marginal distribution (Paul et al., 2005; Zhou and Lange, 2010; Zhou and Zhang, 2012). On the other hand, Newton's method suffers from instability since the log-likelihood functions are non-concave in general.

For distribution fitting, Zhou and Lange (2010) derive stable algorithms based on the minorizationmaximization (MM) principle (Lange et al., 2000). Similar to the classical expectation-maximization algorithm, MM algorithm increases the objective value at each iteration and converges to a stationarity point of objective function under mild regularity conditions.

For regression models, Zhang et al. (2017) propose an iteratively reweighted Poisson regression (IRPR) method for maximum likelihood estimation. Their derivation again hinges upon the MM principle, resulting in the much desirable stability of the IRPR algorithm which is critical as the number of parameters is potentially large.

In practice, MM algorithm may suffer from slow convergence especially in the proximity of the optimum. MGLM organically combines the MM algorithm and the Newton's method. At each iteration, it computes both MM and Newton updates and chooses the one that results in a higher log-likelihood. Thus, stability is ensured as the log-likelihood always increases. When sufficiently close to the optimum, Newton's method takes over and quickly converges to the MLE in just a few iterations.

An added advantage of the MM algorithm is that it separates parameters and embraces parallel computing (Zhou et al., 2010). Each iteration of the IRPR algorithm involves solving $d_{e}$ independent Poisson regressions (Zhang et al., 2017) that can be carried out in parallel. Building upon the parallel package in $\mathrm{R}$, the MGLMreg regression function supports multi-threading on a multi-core machine.

For sparse regression in the MGLMsparsereg function, we implemented the accelerated proximal gradient (Nesterov) method (Zhang et al., 2017). Each iteration only involves evaluation of the gradient of the negative log-likelihood, followed by the elementwise, groupwise, or singular value thresholding according to the regularization being used, and thus scales well with the problem size.

\section{The $\mathbf{R}$ package aspect}

Three main functions of the MGLM package are MGLMfit for fitting multivariate distributions, MGLMreg for fitting multivariate response regressions, and MGLMsparsereg for fitting sparse regressions. The package adopts $\mathrm{S} 4$ object system. In this section, we demonstrate their basic usage using a simulated RNA-seq data set. The $\mathrm{R}$ vignette included in the package provides more extensive examples.

The rnaseq data that comes with the package is simulated from the isoform package (Sun, 2014; Sun et al., 2015) and mimics the real counts generated by the RNA-seq platforms. The simulation mechanism follows the biological process and has nothing to do with the models in Table 1.

In this example, 6 exons are expressed in a gene. Each observation consists of the expression levels (represented by counts) of each exon along with covariates totalReads, treatment, gender, and age of an individual. 200 observations are simulated. In the generative model, exon expression levels are affected by intercept, number of total reads (on log scale), and treatment. Age and gender are unrelated to the exon counts. 


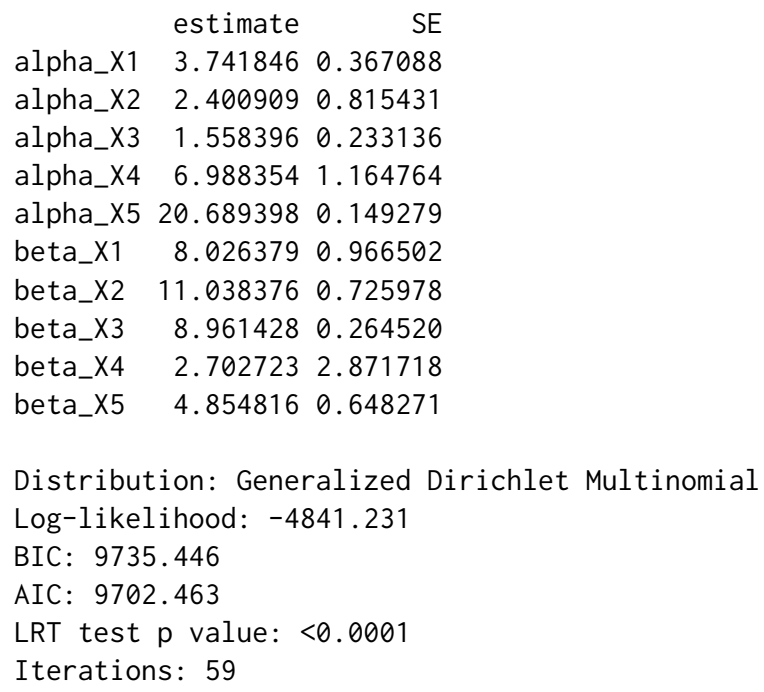

Both dmFit and gdmFit give the LRT $p$-value for comparing the fitted model with the MN model. In this example, both are significantly better than $\mathrm{MN}$, with $p$-values $\ll 0.05$. To compare DM and GDM, we can either compare their BICs or perform a formal LRT.

R> LRT <- -2* (logLik(dmFit) - logLik(gdmFit))

$\mathrm{R}>\operatorname{pchisq}(\mathrm{LRT}, \mathrm{ncol}($ data $)-2$, lower.tail = FALSE)

[1] $5.817352 \mathrm{e}-54$

Both suggest that GDM provides a significantly better fit than DM.

The NegMN model

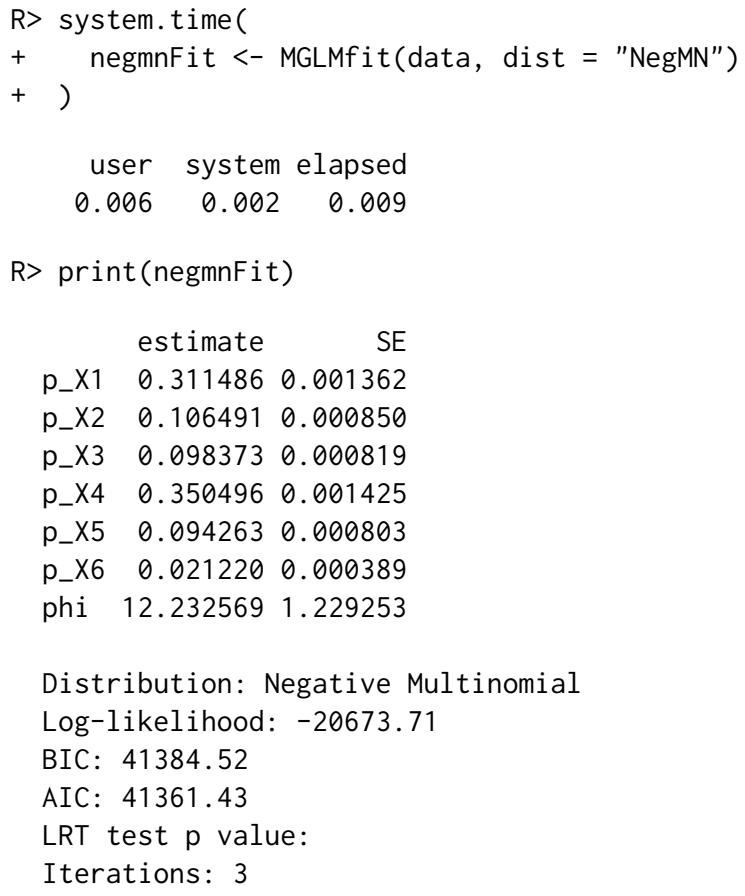

yields a much larger BIC than those of DM and GDM. LRT does not apply here, since NegMN is not a sub-model of the other three.

\section{Regression}

The more interesting question is whether the covariates have a significant relationship to the responses. Regressions are performed using function MGLMreg. First, the regression formula is required by the MGLMreg function. When specifying the regression formula, the response matrix is on the left hand side and the covariates on the right, following the convention in $1 \mathrm{~m}$ and glm. The model is specified 
using the dist argument. The input argument data is optional. When specified, the formula is built based on the supplied data frame; otherwise, the function searches through the global environment for the data elements. Similar to the distribution fitting function, weights of the observations can be specified by the weight argument, and initial values can be supplied using init arguments. Parallel computing is also implemented in the package. Setting parallel=TRUE and giving the number of cores using the argument core invokes multi-threading. The other arguments used to control the algorithm convergence and display include epsilon, maxiters, display, and LRT.

The output of MGLMreg is a list of class "MGLMreg", containing the estimated regression coefficients, their standard errors, Wald test statistics and their corresponding $p$-values for each predictor, loglikelihood, AIC, BIC, gradient and Hessian matrix at the estimate, number of iterations, and fitted values.

We fit the four regression models in Table 1 with all 5 covariates: intercept, number of total reads (on log scale), treatment, age, and gender.

A few observations can be made from the following output. BIC indicates the GDM model as the best fit, followed by the DM model. This is consistent with the distribution fitting results. Note that the hypothesis testing results in the four models are different. In the MN model, all covariates are significant; however, this is not true because age and gender have no effects in the generative model. DM also wrongly identifies age as a significant predictor. Only the GDM model correctly identifies true significant predictors.

\section{MN regression}

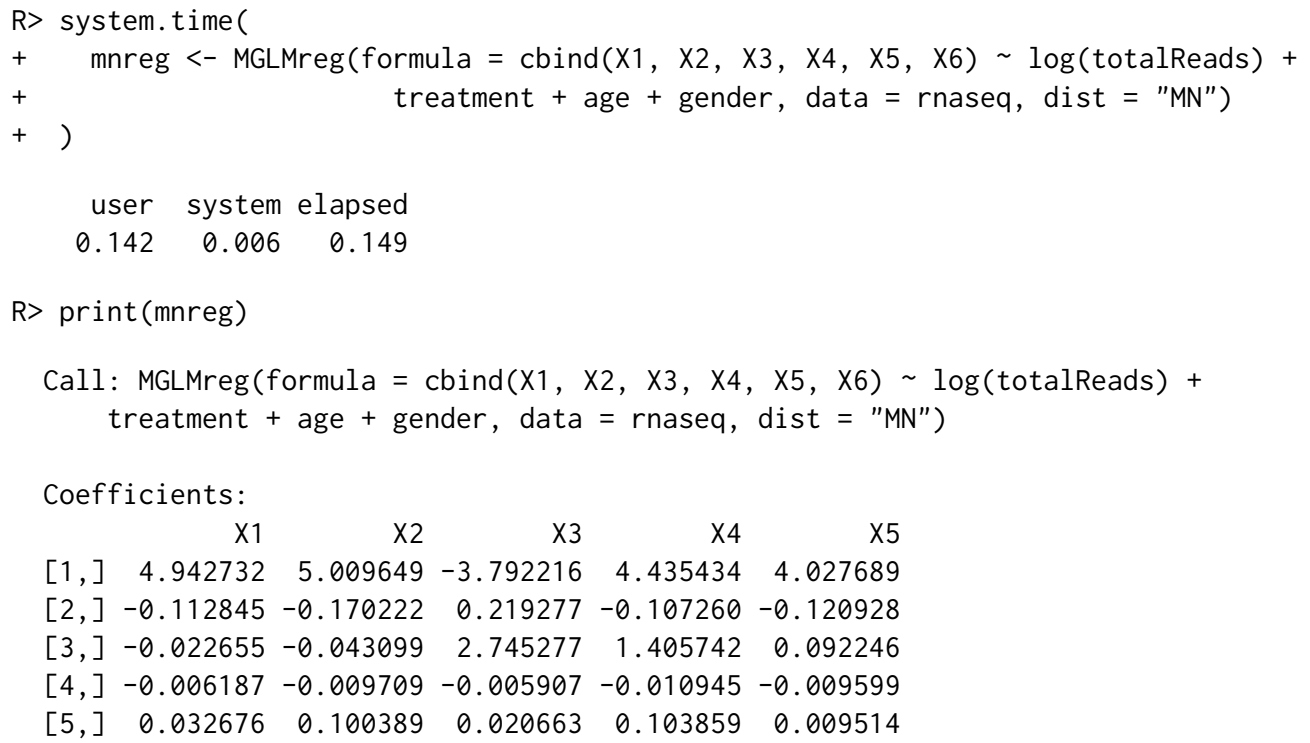

Hypothesis test:

$\begin{array}{lrr} & \text { wald value } & \operatorname{Pr}(>\text { wald }) \\ \text { (Intercept) } & 144.88789 & 1.634268 \mathrm{e}-29 \\ \text { log(totalReads) } & 69.92572 & 1.061922 \mathrm{e}-13 \\ \text { treatment } & 18364.13260 & 0.000000 \mathrm{e}+00 \\ \text { age } & 79.91023 & 8.762650 \mathrm{e}-16 \\ \text { gender } & 52.33670 & 4.601575 \mathrm{e}-10\end{array}$

Distribution: Multinomial

Log-likelihood: -7506.393

BIC: 15145.24

AIC: 15062.79

Iterations: 6

\section{DM regression}

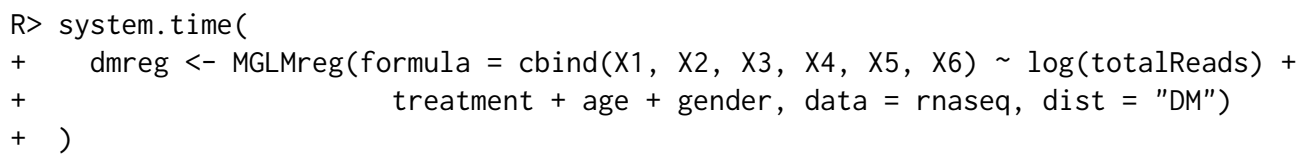


user system elapsed

$\begin{array}{lll}0.182 & 0.004 & 0.187\end{array}$

R> print (dmreg)

Call: MGLMreg (formula $=\operatorname{cbind}(X 1, X 2, X 3, X 4, X 5, X 6) \sim \log ($ totalReads $)+$ treatment + age + gender, data $=$ rnaseq, dist $=$ "DM")

Coefficients:

$\begin{array}{llllll}X 1 & X 2 & X 3 & X 4 & X 5 & X 6\end{array}$

$[1]-,0.895850 \quad-1.096921 \quad-8.997414-1.736871-1.774227 \quad-5.646822$

$\begin{array}{llllllll}{[2,]} & 0.221988 & 0.186919 & 0.536572 & 0.252679 & 0.216672 & 0.347271\end{array}$

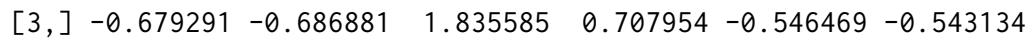

$\begin{array}{llllllll}{[4,]} & 0.010245 & 0.005227 & 0.009134 & 0.004252 & 0.006090 & 0.011642\end{array}$

$\left[\begin{array}{llllllll}{[5,]} & -0.026177 & 0.040244 & -0.052842 & 0.023178 & -0.058339 & -0.039139\end{array}\right.$

Hypothesis test:

$\begin{array}{lrr} & \text { wald value } & \operatorname{Pr}(>\text { wald }) \\ \text { (Intercept) } & 14.579069 & 0.023796 \\ \text { log(totalReads) } & 8.502549 & 0.203547 \\ \text { treatment } & 1851.437449 & 0.000000 \\ \text { age } & 13.131512 & 0.040994 \\ \text { gender } & 4.133364 & 0.658634\end{array}$

Distribution: Dirichlet Multinomial

Log-likelihood: -4386.941

BIC: 8932.831

AIC: 8833.882

Iterations: 9

\section{GDM regression}

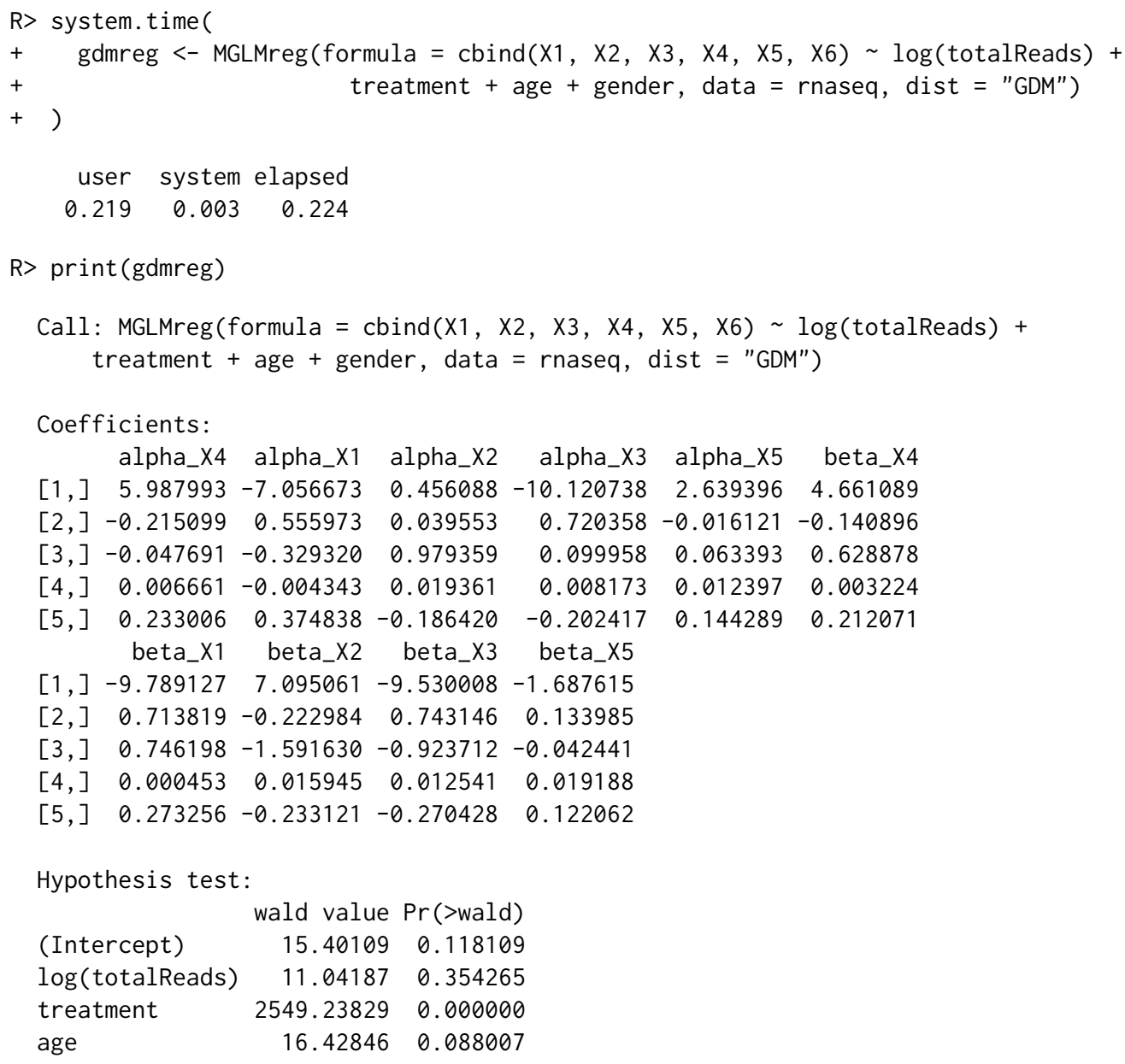




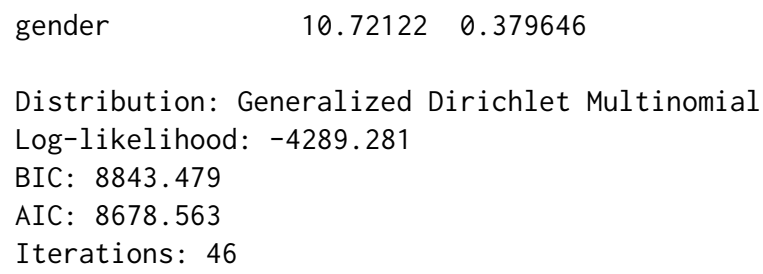

\section{NegMN regression}

There are two variants of NegMN regression, depending on whether to link over-dispersion parameter to covariates. The default setting regBeta=FALSE instructs MGLMreg to fit the NegMN regression with all observations sharing the same over-dispersion parameter value. There are $p d+1$ parameters.
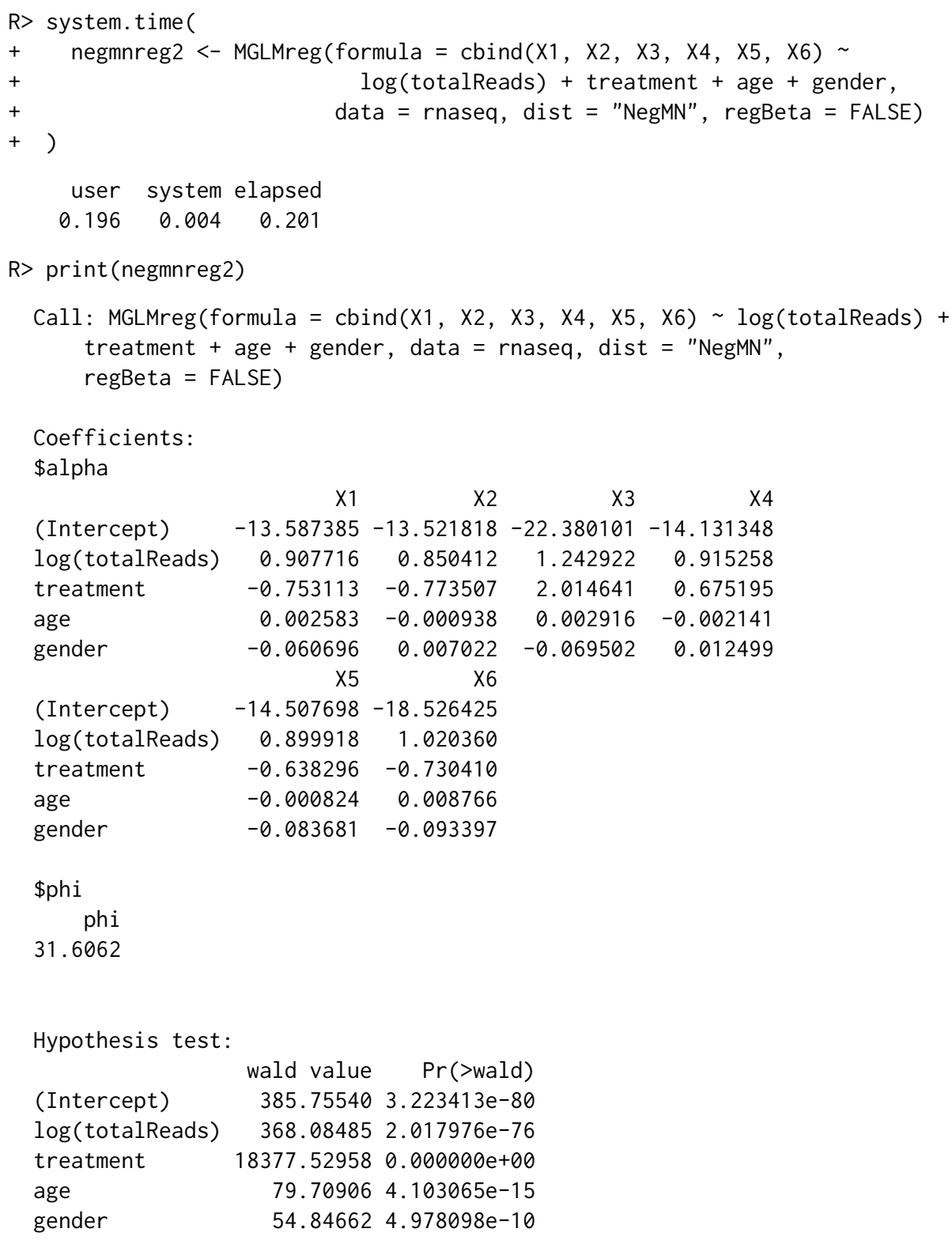

Distribution: Negative Multinomial

Log-likelihood: -8746.689

BIC: 17657.63

AIC: 17555.38

Iterations: 35

regBeta=TRUE instructs MGLMreg to link over-dispersion parameter to covariates and there are $p(d+1)$ regression coefficients. Small likelihood, larger AIC/BIC and slow convergence reflects the lack of fit of this model. 


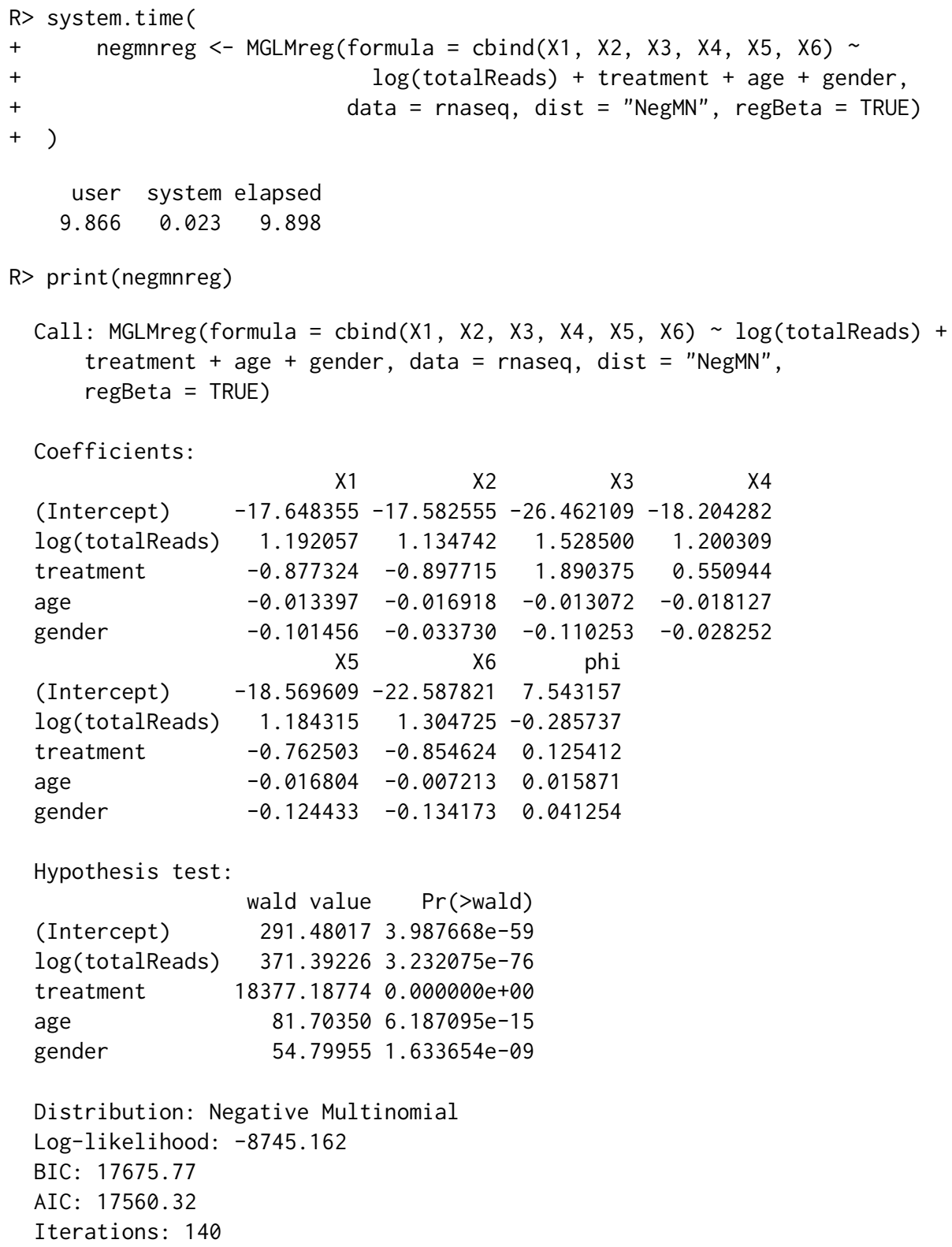

\section{Sparse regression}

The function MGLMsparsereg performs regularized estimation. Similar to MGLMreg, the inputs of the sparse regression function include formula, data, dist, and the convergence controlling arguments. The function also requires the tuning parameter lambda, and the penalty type argument penal ty. The outputs include the coefficient estimates, log-likelihood, AIC, BIC, degrees of freedom, and the number of iterations.

We simulate 100 data points from a 5-variate DM model using 10 covariates. Only three of them (1, 3 , and 5) have non-zero effects. For each 5-variate data point, batch size, or the total number of objects that are put into 5 categories, is drawn from $\operatorname{Bin}(200,0.8)$.

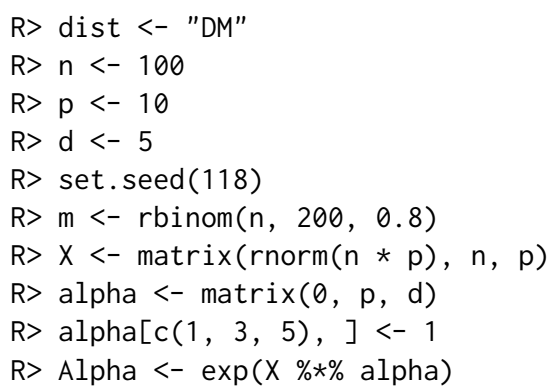


$\mathrm{R}>\mathrm{Y}<-\operatorname{rdirmn}($ size $=\mathrm{m}$, alpha $=$ Alpha $)$

$\mathrm{R}>$ length $(\mathrm{m})$

[1] 100

$\mathrm{R}>\operatorname{head}(\mathrm{m})$

[1] $167 \quad 160162 \quad 159156 \quad 157$

$R>\operatorname{head}(Y)$

$[, 1][, 2][, 3][, 4][, 5]$

$\begin{array}{llllll}{[1,]} & 24 & 7 & 112 & 15 & 9\end{array}$

$[2] \quad 34 \quad 33 \quad 31 \quad 38 \quad$,

$[3] \quad 0 \quad$,

$\begin{array}{llllll}{[4,]} & 7 & 17 & 84 & 29 & 22\end{array}$

$[5] \quad 0 \quad 33 \quad 0 \quad 123 \quad$,

$[6] \quad 0 \quad 0003154 \quad$,

$\mathrm{R}>\operatorname{head}(\operatorname{rowSums}(\mathrm{Y}))$

[1] $\begin{array}{llllll}167 & 160 & 162 & 159 & 156 & 157\end{array}$

We demonstrate the regularized estimation by group, nuclear norm, and element-wise penalization.

\section{Variable selection by group penalty}

With input lambda=Inf, MGLMspar sereg returns $\lambda_{\max }$, the maximum value the tuning parameter can take such that not all regression coefficient estimates are 0 .

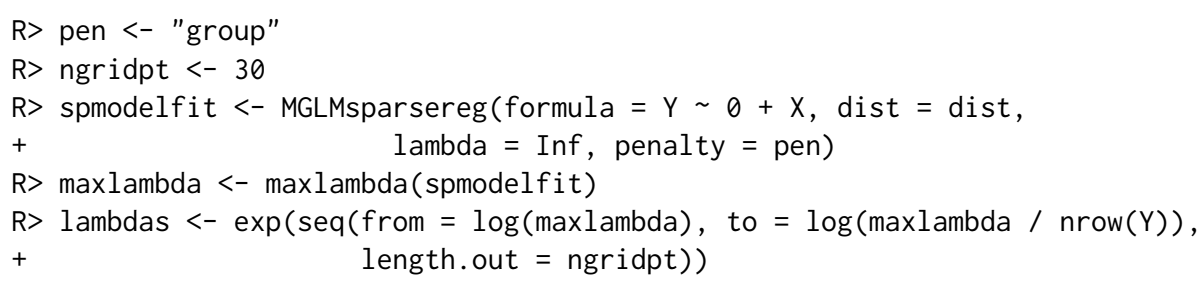

Tuning is performed on 30 grid points. The left panel of Figure 1 displays the BIC trace along the solution path.

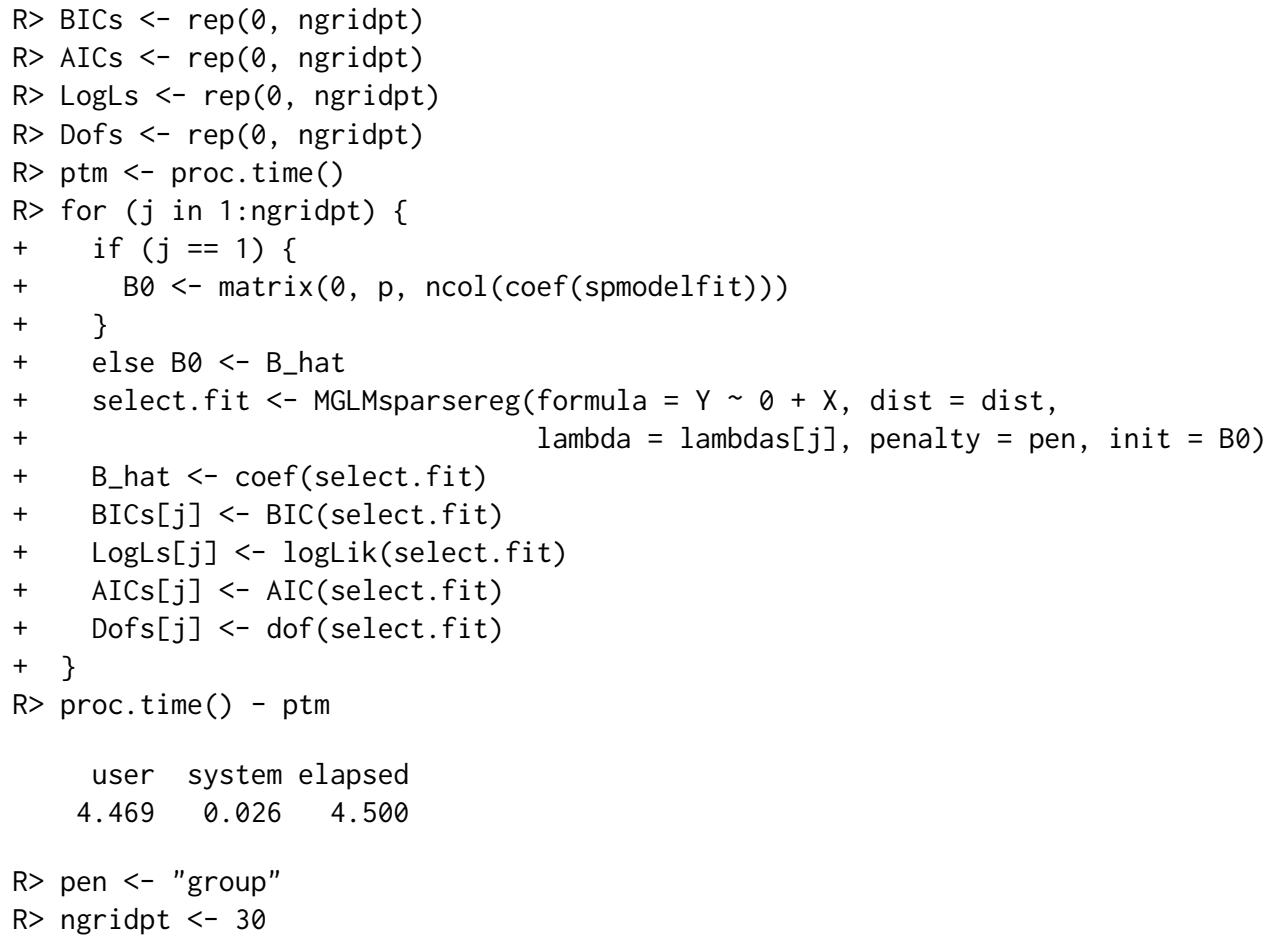



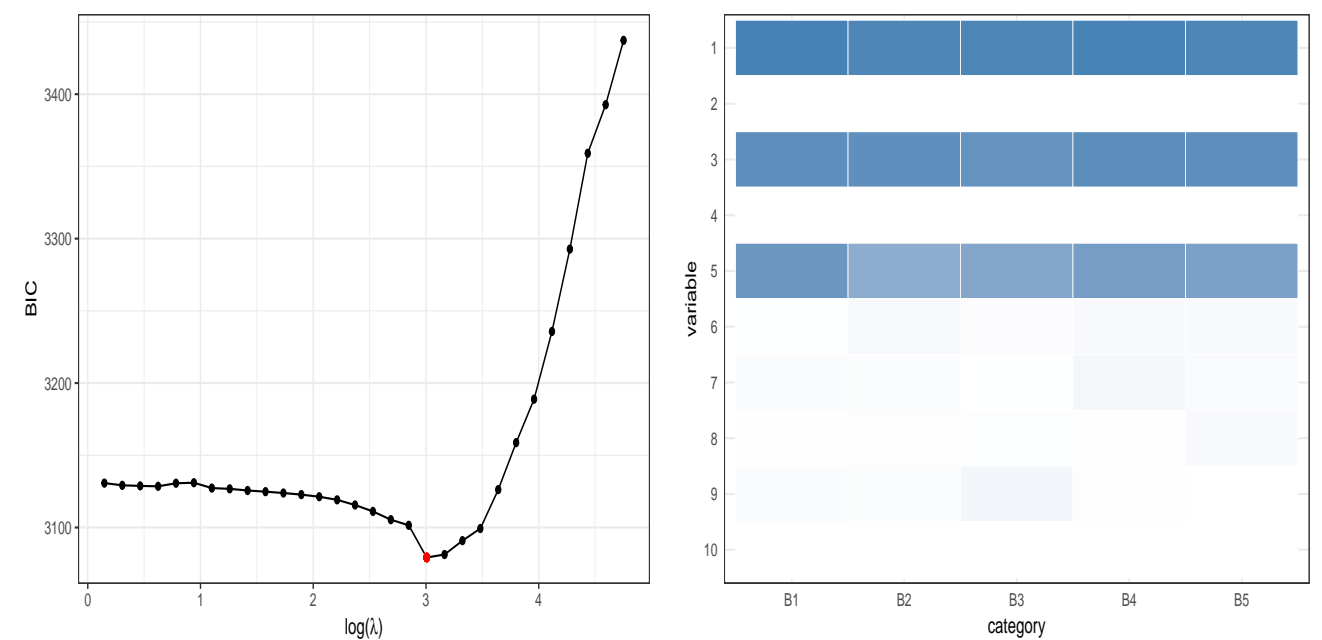

Figure 1: Variable selection by the group penalty. Left: BIC trace. Right: Regularized estimate $\widehat{\boldsymbol{B}}(\lambda)$ at the optimal $\lambda$ displayed in gray scale.

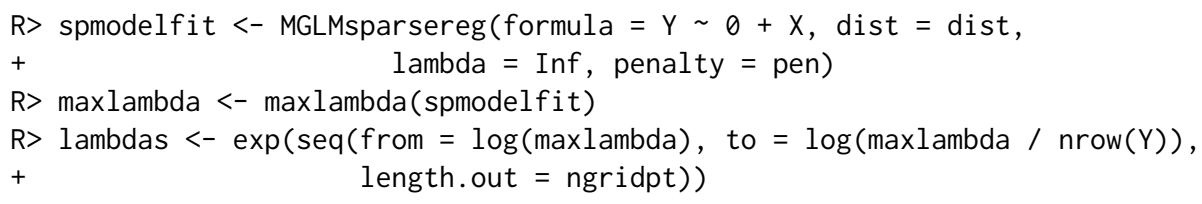

The right panel of Figure 1 displays the regularized estimate $\widehat{B}(\lambda)$ at the tuning parameter value with minimal BIC.

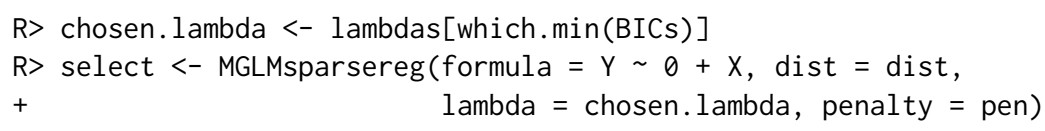

Alternatively, the function MGLMtune automates the tuning procedure and reports the regularized estimate at the optimal tuning parameter value according to BIC.

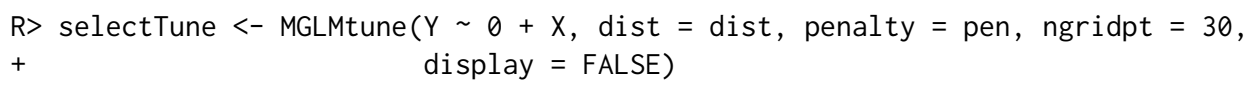

The option ngridpt sets the number of grid points $n_{\text {grid }}$ and the sequence of tuning parameters is equally spaced on the $\log$ scale within the interval $\left[\lambda_{\max } / n_{\text {grid }}, \lambda_{\max }\right]$.

\section{Low rank regression by nuclear norm regularization}

Nuclear norm regularization is invoked by setting penalty="nuclear".

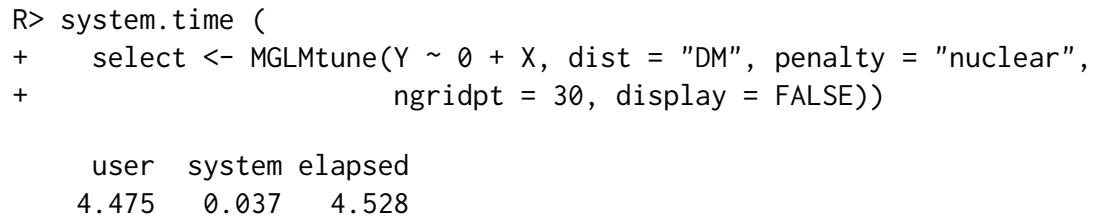

Figure 2 displays the BIC trace plot and the regularized estimate at optimal $\lambda$ from the same data by the nuclear norm penalty.

\section{Select by entries}

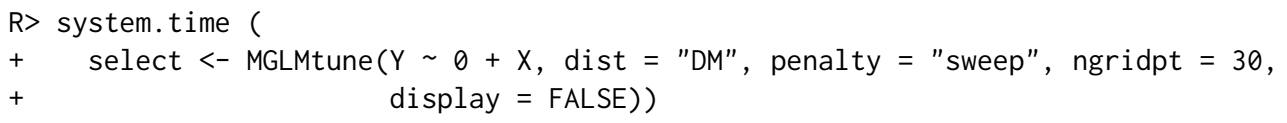



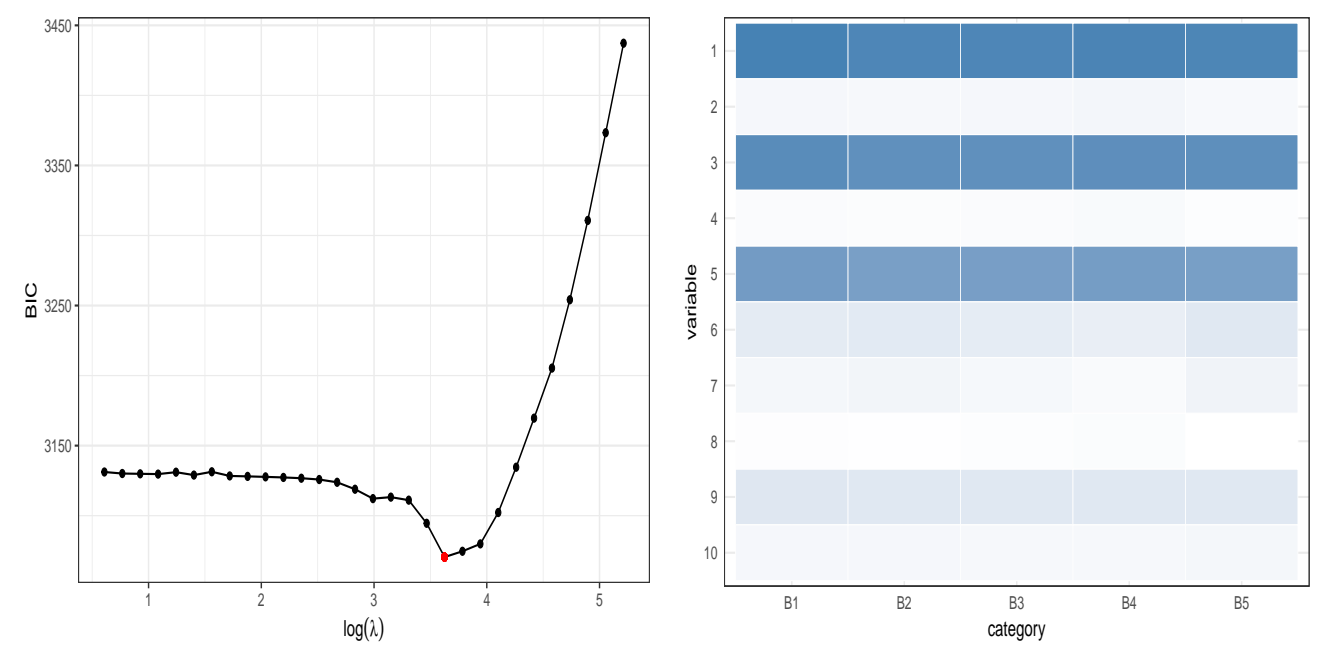

Figure 2: Low rank regression by the nuclear norm penalty. Left: BIC trace. Right: Regularized estimate $\widehat{\boldsymbol{B}}(\lambda)$ at the optimal $\lambda$ displayed in gray scale.
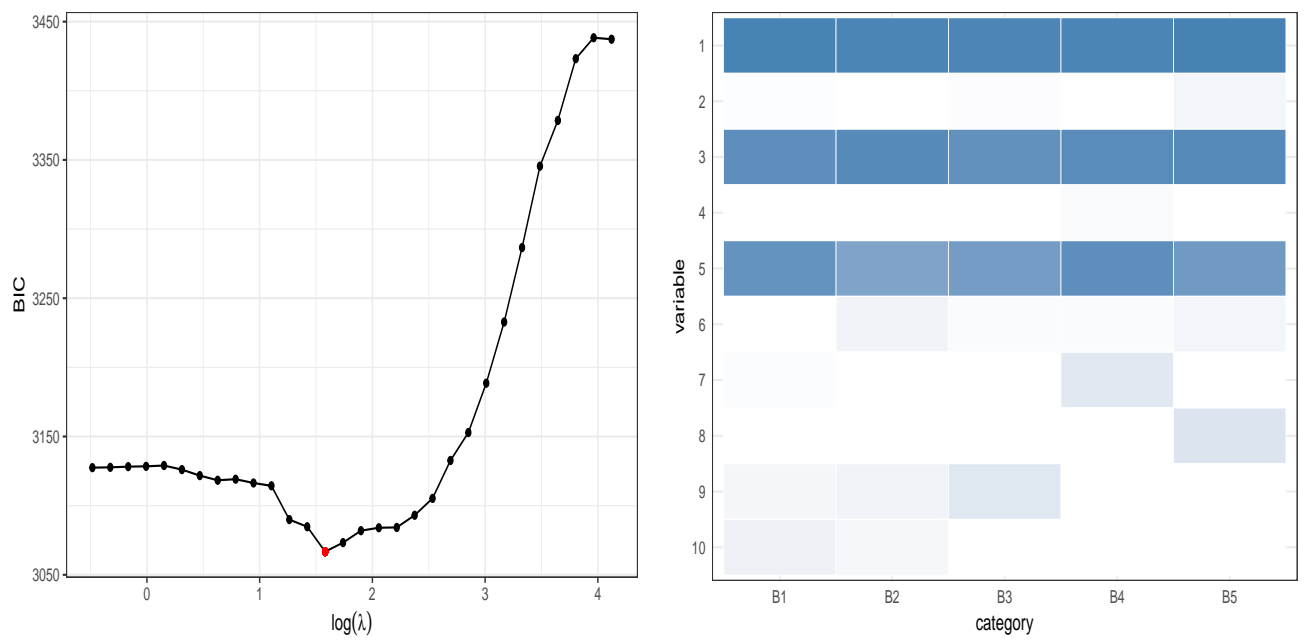

Figure 3: Select by parameter matrix entries with L1 norm penalty. Left: BIC trace. Right: Regularized estimate $\widehat{B}(\lambda)$ at the optimal $\lambda$ displayed in gray scale.
user system elapsed
$4.448 \quad 0.038 \quad 4.504$

Figure 3 displays the BIC trace plot and the regularized estimate at optimal $\lambda$ from the same data by the element-wise lasso penalty.

\section{Discussion}

This article introduces the MGLM package for analysis of multivariate categorical data. Distribution fitting, regression, sparse regression, and random number generation are implemented in a simple and unified framework. It timely responds to the current challenge of multivariate categorical data analysis arising from modern technology such as RNA-seq. The R package is available on CRAN.

There are several possible extensions that would be useful to the package. Some of them include:

1. Alternative parameterization. Some models in Table 1 admits alternative parameterization. For 
instance, the DM distribution can be re-parameterized as

$$
\theta=\frac{1}{\sum_{j} \alpha_{j}}, p_{j}=\frac{\alpha_{j}}{\sum_{j^{\prime}} \alpha_{j^{\prime}}}, j=1, \ldots, d
$$

in terms of the proportion vector $\boldsymbol{p}=\left(p_{1}, \ldots, p_{d}\right) \in \Delta_{d}$ and the overdispersion parameter $\theta>0$. Appropriate inverse link function can be

$$
\begin{aligned}
p_{j} & =\frac{e^{\mathbf{x}^{T} \boldsymbol{\beta}_{j}}}{1+\sum_{j^{\prime}=1}^{d-1} e^{\mathbf{x}^{T} \boldsymbol{\beta}_{j^{\prime}}}}, \quad j=1, \ldots, d-1, \\
\theta & =e^{\mathbf{x}^{T} \boldsymbol{\beta}_{d} .}
\end{aligned}
$$

The multinomial-logit model can be treated as a special case with $\boldsymbol{\beta}_{d}=\mathbf{0}$. Zhou and Lange (2010) discuss an algorithm for distribution fitting using this parameterization. Corresponding estimation algorithm for the regression model needs to be devised and implemented. Similar re-parameterization applies to the GDM model.

2. Alternative link function. Current version only implements the log link function for positive distribution parameter and logit link for the probability vector. Inclusion of alternative link functions such as probit and cloglog would expand the flexibility of the models.

3. Ordinal categorical responses. Multinomial-logit model can be adapted to ordinal categorical responses (Agresti, 2002, Chapter 7). Parallel developments and implementation for the DM, NegMN, and GDM models are worth considering.

4. Parameter constraints. Current version does not allow constraints among regression parameters. MGLMreg calls glm. fit functions to fit weighted Poisson regressions in each iteration; we may call functions from glmc package (Chaudhuri et al., 2006) instead to incorporate parameter constraints.

5. The xij argument (Yee, 2015, Chapter 14). Current version assumes the same set of covariates for all categories. Allowing covariates to take different values for each category can be useful, e.g., for discrete choice modeling in econometrics. The corresponding algorithm and implementation are worth exploring.

The MGLM package for $\mathrm{R}$ is continually being developed.

\section{Acknowledgments}

The work is partially supported by NSF grants DMS-1127914, DMS-1310319 and NIH grants HG006139, GM105785 and GM53275.

\section{Bibliography}

A. Agresti. Categorical Data Analysis. Wiley Series in Probability and Statistics. John Wiley \& Sons, New York, 2nd edition, 2002. ISBN 0-471-36093-7. [p73, 88]

S. G. Baker. The multinomial-poisson transformation. Journal of the Royal Statistical Society. Series D (The Statistician), 43(4):pp. 495-504, 1994. ISSN 00390526. URL http://www. jstor. org/stable/2348134. [p74]

N. Bouguila. Clustering of count data using generalized dirichlet multinomial distributions. IEEE Transactions on Knowledge and Data Engineering, 20(4):462-474, 2008. ISSN 1041-4347. URL http: //doi.org/10.1109/TKDE.2007.190726. [p76]

S. Boyd and L. Vandenberghe. Convex Optimization. Cambridge University Press, Cambridge, 2004. ISBN 0-521-83378-7. [p74]

S. Chaudhuri, M. S. Handcock, and M. S. Rendall. Glmc: An R Package for Generalized Linear Models Subject to Constraints, 2006. URL http://www. stat. washington. edu/handcock/combining. [p88]

R. J. Connor and J. E. Mosimann. Concepts of independence for proportions with a generalization of the Dirichlet distribution. J. Amer. Statist. Assoc., 64:194-206, 1969. ISSN 0162-1459. [p76]

B. Efron and D. V. Hinkley. Assessing the accuracy of the maximum likelihood estimator: Observed versus expected Fisher information. Biometrika, 65(3):457-487, 1978. ISSN 0006-3444. URL http: //doi.org/10.1093/biomet/65.3.457. [p77] 
J. Fan and R. Li. Variable selection via nonconcave penalized likelihood and its oracle properties. J. Amer. Statist. Assoc., 96(456):1348-1360, 2001. ISSN 0162-1459. URL http://doi .org/10.1198/ 016214501753382273. [p77]

I. E. Frank and J. H. Friedman. A statistical view of some chemometrics regression tools. Technometrics, 35(2):109-135, 1993. ISSN 00401706. URL http://doi .org/10.2307/1269656. [p77]

J. H. Friedman, T. Hastie, and R. Tibshirani. Regularization paths for generalized linear models via coordinate descent. Journal of Statistical Software, 33(1):1-22, 2010. ISSN 1548-7660. URL http://www. jstatsoft.org/v33/i01. [p73,77]

R. L. Graham, D. E. Knuth, and O. Patashnik. Concrete Mathematics. Addison-Wesley Publishing Company, Reading, MA, 2nd edition, 1994. ISBN 0-201-55802-5. A foundation for computer science. [p75]

J. B. Lang. On the comparison of multinomial and Poisson log-linear models. J. Roy. Statist. Soc. Ser. B, 58 (1):253-266, 1996. ISSN 0035-9246. URL http://links. jstor. org/sici?sici=0035-9246(1996)58: 1<253: OTCOMA>2.0. C0;2-P\&origin=MSN. [p74]

K. Lange, D. R. Hunter, and I. Yang. Optimization transfer using surrogate objective functions. J. Comput. Graph. Statist., 9(1):1-59, 2000. ISSN 1061-8600. With discussion, and a rejoinder by Hunter and Lange. [p78]

R. Mazumder, T. Hastie, and R. Tibshirani. Spectral regularization algorithms for learning large incomplete matrices. Journal of Machine Learning Research, 11:2287-2322, 2010. [p78]

L. Meier, S. van de Geer, and P. Bühlmann. The group Lasso for logistic regression. J. R. Stat. Soc. Ser. B Stat. Methodol., 70(1):53-71, 2008. ISSN 1369-7412. [p77]

S. R. Paul, U. Balasooriya, and T. Banerjee. Fisher information matrix of the Dirichlet-multinomial distribution. Biom. J., 47(2):230-236, 2005. ISSN 0323-3847. [p78]

S. Powers, T. Hastie, and R. Tibshirani. Nuclear penalized multinomial regression with an application to predicting at-bat outcomes in baseball. To appear in special edition "Statistical Modelling for Sports Analytics", Statistical Modelling. [p78]

W. Sun. Isoform: A Set of Tools for RNA Isoform Study Using RNA-Seq Data, 2014. URL http: //www. bios . unc. edu/ weisun/software/isoform. htm. R package version 0.99.2. [p78]

W. Sun, Y. Liu, J. J. Crowley, and et al. IsoDOT detects differential RNA-isoform expression/usage with respect to a categorical or continuous covariate with high sensitivity and specificity. J. Amer. Statist. Assoc., 110(511):975-986, 2015. ISSN 0162-1459. URL http://doi .org/10. 1080/01621459. 2015. 1040880. [p78]

R. Tibshirani. Regression shrinkage and selection via the lasso. J. Roy. Statist. Soc. Ser. B, 58(1):267288, 1996. ISSN 0035-9246. URL http://links. jstor.org/sici?sici=0035-9246(1996) 58: $1<267$ : RSASVT>2.0.CO;2-G\&origin=MSN. [p77]

T. Tvedebrink. Overdispersion in allelic counts and $\theta$-correction in forensic genetics. Theoretical Population Biology, 78(3):200-210, 2010. ISSN 0040-5809. URL http://doi.org/10.1016/j. tpb. 2010.07.002. [p73]

W. N. Venables and B. D. Ripley. Modern Applied Statistics with S. Springer-Verlag, New York, 4th edition, 2002. ISBN 0-387-95457-0. URL http://www. stats.ox.ac.uk/pub/MASS4. [p73]

T. W. Yee. The VGAM package for categorical data analysis. Journal of Statistical Software, 32(10):1-34, 2010. ISSN 1548-7660. URL http://www. jstatsoft.org/v32/i10. [p73]

T. W. Yee. Vector Generalized Linear and Additive Models: With an Implementation in R. Springer-Verlag, New York, USA, 2015. [p73, 88]

T. W. Yee. VGAM: Vector Generalized Linear and Additive Models, 2017. URL https: //CRAN . R-project. org/package=VGAM. R package version 1.0-4. [p73]

M. Yuan and Y. Lin. Model selection and estimation in regression with grouped variables. J. R. Stat. Soc. Ser. B Stat. Methodol., 68(1):49-67, 2006. ISSN 1369-7412. URL http://doi .org/10.1111/j.14679868.2005.00532.x. [p77]

C.-H. Zhang. Nearly unbiased variable selection under minimax concave penalty. Ann. Statist., 38(2): 894-942, 2010. ISSN 0090-5364. URL http://doi .org/10.1214/09-A0S729. [p77] 
Y. Zhang, H. Zhou, J. Zhou, and W. Sun. Regression models for multivariate count data. J. Comput. Graph. Statist., 26(1):1-13, 2017. ISSN 1061-8600. URL http://doi.org/10. 1080/10618600. 2016. 1154063. [p78]

H. Zhou and K. L. Lange. MM algorithms for some discrete multivariate distributions. Journal of Computational and Graphical Statistics, 19:645-665, 2010. [p76, 78, 88]

H. Zhou and L. Li. Regularized matrix regressions. Journal of Royal Statistical Society, Series B, 76(2): 463-483, 2014. [p78]

H. Zhou and Y. Zhang. EM vs MM: a case study. Computational Statistics E Data Analysis, 56:3909-3920, 2012. [p78]

H. Zhou, K. L. Lange, and M. A. Suchard. Graphical processing units and high-dimensional optimization. Statistical Science, 25:311-324, 2010. [p78]

H. Zou and T. Hastie. Regularization and variable selection via the elastic net. J. R. Stat. Soc. Ser. B Stat. Methodol., 67(2):301-320, 2005. ISSN 1369-7412. URL http://doi.org/10.1111/j.14679868.2005.00503.x. [p77]

\author{
Juhyun Kim \\ Department of Biostatistics \\ University of California, Los Angeles \\ juhkim111@ucla.edu \\ Yiwen Zhang \\ KPMG LLP \\ Irvine, $C A$ \\ zhangyiwen1015@gmail.com \\ Joshua Day \\ Department of Statistics \\ North Carolina State University \\ jtdayzencsu.edu \\ Hua Zhou \\ Department of Biostatistics \\ University of California, Los Angeles \\ huazhou@ucla.edu
}

